\title{
Effectiveness of BNT162b2 mRNA vaccine and ChAdOx1 adenovirus vector vaccine on mortality following COVID-19
}

Dr Jamie Lopez Bernal ${ }^{1,2,3}$ (Consultant Medical Epidemiologist), Prof Nick Andrews $s^{1,2}$ (Senior Statistician), Dr Charlotte Gower ${ }^{1}$ (Senior Epidemiologist), Dr Julia Stowe ${ }^{1}$ (Senior Epidemiologist), Elise Tessier ${ }^{1}$ (Senior Epidemiologist), Dr Ruth Simmons ${ }^{1}$ (Senior Epidemiologist), Prof Mary Ramsay $^{1,2}$ (Head of Immunisations)

1. Public Health England, London, United Kingdom

2. NIHR Health Protection Research Unit in Vaccines and Immunisation, London School of Hygiene and Tropical Medicine, London, United Kingdom

3. NIHR Health Protection Research Unit in Respiratory Infections, Imperial College London, United Kingdom

Corresponding author: jamie.lopezbernal2@phe.gov.uk; 61 Colindale Ave, London, NW9 5EQ, UK; ORCID: 0000-0002-1301-5653

\section{Abstract}

We estimated risk of death in vaccinated compared to unvaccinated COVID-19 cases. Cases vaccinated with 1 dose of BNT162 b2 had $44 \%$ reduced risk of death, $55 \%$ with 1 dose of ChAdOx1, and $69 \%$ with 2 doses of BNT162 b2. This is on top of the protection provided against becoming a case.

\section{Introduction}

Real world effectiveness data has begun to emerge for COVID-19 vaccines, showing high levels of protection against both symptomatic and asymptomatic infection, supporting the findings of the phase III clinical trials.(1-6) Nevertheless, evidence on effectiveness against mortality, the most severe outcome, is currently limited and has not yet been reported for most vaccines.(2)

The UK was the first country to implement a COVID-19 vaccination programme, starting with the Pfizer-BioNTech BNT162b2 mRNA vaccine in December and followed soon afterwards with the Oxford-Astrazeneca ChAdOx1 adenovirus vector vaccine. Vaccination was offered to older adults, care home residents and health and social care workers first, delivery was subsequently rolled out to increasingly younger age cohorts and to clinical risk groups. A policy decision was made early on in the programme to use an extended dosing interval of 12 weeks, in order to maximise the number of vulnerable people offered the first dose.

Early data suggested that a single dose of BNT162 b2 was $80-85 \%$ effective at preventing mortality in individuals aged over 80 years. (2) However, effectiveness of ChAdOx1 against mortality has not yet been reported. 
medRxiv preprint doi: https://doi.org/10.1101/2021.05.14.21257218; this version posted May 18, 2021. The copyright holder for this preprint

(which was not certified by peer review) is the author/funder, who has granted medRxiv a license to display the preprint in perpetuity.

It is made available under a CC-BY-NC-ND 4.0 International license .

\section{Methods}

This study estimates the risk of death among confirmed cases of COVID-19 according to their vaccination status.

In England a community COVID-19 testing programme is available to individuals reporting symptoms (high temperature, new continuous cough, loss or change in sense of smell or taste), care home residents and staff, and individuals taking part in local or national mass asymptomatic testing. We linked all new symptomatic PCR positive cases to mortality data from NHS records.(7) Testing and deaths data between $8^{\text {th }}$ December (when the vaccination programme started) and $17^{\text {th }}$ April were initially extracted. This was subsequently restricted to deaths to April $6^{\text {th }}$ as lags in deaths registrations could affect the most recent days.

Survival analysis was conducted to estimate the hazard ratios for death within 28 days of a positive SARS-CoV-2 PCR test by vaccination status: unvaccinated, vaccinated with one dose (where the first dose was received at least 21 days before the test date), vaccinated with 2 doses (where the second dose was received at least 7 days before). We also estimated effects among those who received first doses 0-20 days prior to testing positive (earlier than an effect would be anticipated) as a control analysis. Adjustments were made for sex, clinical risk factors for severe disease (including immunosuppressive disease or therapy, severe respiratory disease, rare diseases and inborn errors of metabolism that significantly increase the risk of infections, pregnancy with significant congenital heart disease),(8) age in 5 year bands, and whether they had been identified as a care home resident. Estimates were stratified by age group (70-79 and 80+ years) and whether they were a care home resident.

\section{Results}

48,096 cases aged 70 years and above were included in the analysis, of which $79.1 \%$ were unvaccinated; $12.7 \%$ had been vaccinated with BNT162b2 (3,910 received their first dose within 20 days of their test date, 2,007 received their first dose $>=21$ days before their test date and 191 received their $2^{\text {nd }}$ dose $>=7$ days before their test date); and $8.2 \%$ had been vaccinated with ChAdOx1 (2,686 received their first dose within 20 days of their test date, 1,258 received their first dose $>=21$ days before their test date and 6 received their $2^{\text {nd }}$ dose $>=7$ days before their test date).

Kaplan Meier curves showing the proportion who died by days after the positive test for the first dose and unvaccinated are shown in figure 1 . Among $80+$ year olds with at least 28 days of follow-up data $16.1 \%(1462 / 9105)$ of unvaccinated cases died, $9.2 \%(99 / 1,072)$ of cases vaccinated with 1 dose of BNT162b2 died, 11.3\% (33/293) of cases vaccinated with 1 dose of ChAdOx1, and 4.7\% (6/128) of cases vaccinated with 2 doses of BNT162b2 died. Among 70-79 year olds with at least 28 days of follow-up data $4.0 \%(1,147 / 28,875)$ of unvaccinated cases died, $2.7 \%(15 / 549)$ of cases vaccinated with 1 dose of BNT162b2 died, 2.1\% (10/484) of cases vaccinated with 1 dose of ChAdOx1, and $0 \%$ $(0 / 7)$ of cases vaccinated with 2 doses of BNT162b2 died. There were no individuals with 28 days of follow-up after 2 doses of ChAdOx1. 

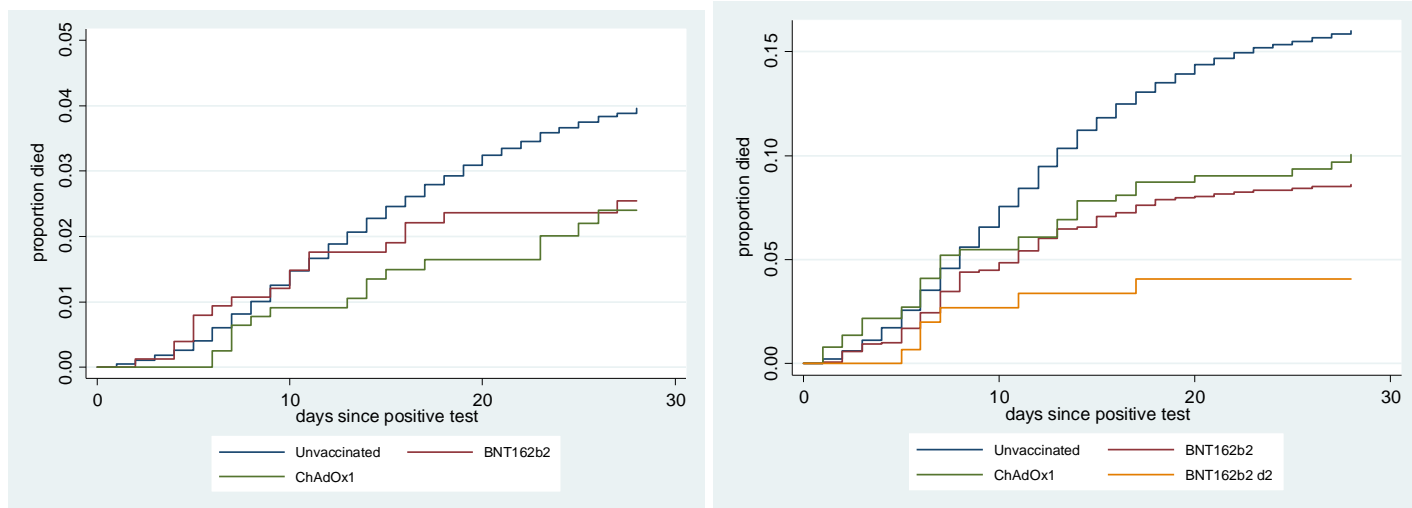

Figure 1: Proportion died by vaccination status (a) 70-79 year olds (b) 80+ year olds

In the fully adjusted model, hazard ratios compared to unvaccinated cases were $0.56(95 \% \mathrm{Cl} 0.47$ $0.68)$ for 1 dose of BNT162b2, 0.45 (95\% Cl 0.34-0.59) for 1 dose of ChAdOx 1 and 0.31 (95\%Cl 0.14 $0.69)$ for 2 doses of BNT162 b2. This indicates an additional $44 \%$ protection against death after the first dose of BNT162b2, 55\% after the first dose of ChAdOx1, and $69 \%$ after the second dose of BNT162b2, on top of the protection against become a case. There was little difference by age group; the hazard ratios after the first BNT162b2 dose were 0.49 (95\% $\mathrm{Cl} 0.31-0.78)$ and $0.56(95 \% \mathrm{Cl} 0.46-$ 0.69 ) for age 70-79 and 80+, respectively, whilst for ChAdOx1 they were $0.46(95 \% \mathrm{Cl} 0.28-0.75)$ and $0.45(95 \% \mathrm{Cl} 0.32-0.63)$ respectively. Note that in age $80+$ care home adjustment was important and led to a lower hazard ratio for ChAdOx1 despite the crude Kaplan-Meier showing lower mortality for BNT162b2.

In the control analysis among those vaccinated with first doses $0-20$ days prior to testing positive, the hazard ratio with BNT162b2 was 0.81 (95\% 0.73-0.91) and with ChAdOx1 0.99 (95\% Cl 0.87-1.12).

There were notable differences by care home resident status. Among care home residents, hazard ratios were 0.87 (95\% Cl 0.60-1.25) for 1 dose of BNT162b2 and 0.37 (95\% $\mathrm{Cl} 0.23-0.60)$ for 1 dose of ChAdOx1 (numbers were too small for estimating 2 dose effects with either vaccine). Among noncare home residents, hazard ratios compared to unvaccinated cases were 0.48 ( $95 \% \mathrm{Cl} 0.38-0.59)$ for 1 dose of BNT162b2 and $0.52(95 \% \mathrm{Cl} 0.37-0.73)$ for 1 dose of ChAdOx1.

\section{Discussion}

We found that confirmed cases of COVID-19 who had been vaccinated with either a single dose of BNT162b2 or a single dose of ChAdOx1 had significantly reduced risk of dying compared to unvaccinated cases. The point estimates showed greater protection with ChAdOx1, though confidence intervals overlapped suggesting little difference between the two vaccines. Combined with recent estimates of protection against symptomatic disease in the same age group, these findings suggest that a single dose of either vaccine is approximately $80 \%$ effective at preventing mortality, and two doses of BNT162b2 is approximately $97 \%$ effective at preventing mortality in older adults. $(2,9)$

The phase three clinical trials have been unable to report on efficacy against mortality due to smaller numbers. Our findings with BNT162b2 were similar to real world findings previously reported in the UK and Israel, both in the context of B.1.1.7 variant as the dominant strain. $(2,10)$ Our study is the 
medRxiv preprint doi: https://doi.org/10.1101/2021.05.14.21257218; this version posted May 18, 2021. The copyright holder for this preprint

(which was not certified by peer review) is the author/funder, who has granted medRxiv a license to display the preprint in perpetuity.

It is made available under a CC-BY-NC-ND 4.0 International license .

first to estimate for the effectiveness of ChAdOx1 against mortality and suggests that a single dose offers similar levels of protection to BNT162b2.

We found that although both vaccines provided additional protection against mortality overall, among care home residents who received a single dose of the Pfizer vaccine, the effect was small and non-significant suggesting that there was no clear evidence additional protection (beyond the protection against becoming a case). Though this could be due to residual confounding and lacking statistical power in this group.

Our study is observational and as such we are unable to exclude unmeasured or residual confounding. For example, healthy vaccinee effects, whereby healthier individuals are more likely to present for vaccination and also more likely to survive, could be an issue. We see some evidence of a possible healthy vaccinee effect with the BNT162b2 because we see a small effect in days 0-20 after the first dose, before a vaccine effect might be anticipated, whereas we don't see this effect with ChAdOx1. However, we have previously found that vaccine effects occur more rapidly with BNT162b2, so this could represent early vaccine effects.(2) A further limitation is that some of the deaths within 28 days of a positive test may be unrelated to COVID and therefore not preventable by vaccination. All of the individuals included in this analysis had reported symptoms of COVID-19, therefore the proportion of deaths unrelated to COVID is likely to be small, however, this could cause us to underestimate the effects on mortality.

In conclusion, we find strong evidence that both vaccines offer high levels of protection against mortality after a single dose which supports prioritising the first dose in at risk groups in the context of high disease incidence and vaccine supply or delivery constraints. Nevertheless, a second dose of BNT162b2 offers yet further protection against mortality, highlighting the importance of completing the course. We are not yet able to estimate two dose effects with ChAdOx1 due to the later rollout of this vaccine. 
medRxiv preprint doi: https://doi.org/10.1101/2021.05.14.21257218; this version posted May 18, 2021. The copyright holder for this preprint

(which was not certified by peer review) is the author/funder, who has granted medRxiv a license to display the preprint in perpetuity.

It is made available under a CC-BY-NC-ND 4.0 International license .

\section{References}

1. Hall VJ, Foulkes S, Saei A, Andrews N, Oguti B, Charlett A, et al.

Effectiveness of BNT162b2 mRNA vaccine against infection and COVID-19 vaccine coverage in healthcare workers in England, multicentre prospective cohort study (the SIREN study). 2021.

2. Lopez Bernal J, Andrews N, Gower C, Robertson C, Stowe J, Tessier

$\mathrm{E}$, et al. Effectiveness of the Pfizer-BioNTech and Oxford-AstraZeneca vaccines on covid-19 related symptoms, hospital admissions, and mortality in older adults in England: test negative case-control study. BMJ.

2021;373:n1088.

3. Shrotri M, Krutikov M, Palmer T, Giddings R, Azmi B, Subbarao S, et al. Vaccine effectiveness of the first dose of ChAdOx1 nCoV-19 and BNT162b2 against SARS-CoV-2 infection in residents of Long-Term Care Facilities (VIVALDI study). medRxiv. 2021:2021.03.26.21254391.

4. Dagan N, Barda N, Kepten E, Miron O, Perchik S, Katz MA, et al. BNT162b2 mRNA Covid-19 Vaccine in a Nationwide Mass Vaccination Setting. The New England journal of medicine. 2021.

5. Voysey M, Clemens SAC, Madhi SA, Weckx LY, Folegatti PM, Aley PK, et al. Safety and efficacy of the ChAdOx1 nCoV-19 vaccine (AZD1222) against SARS-CoV-2: an interim analysis of four randomised controlled trials in Brazil, South Africa, and the UK. The Lancet. 2020.

6. Polack FP, Thomas SJ, Kitchin N, Absalon J, Gurtman A, Lockhart S, et al. Safety and Efficacy of the BNT162b2 mRNA Covid-19 Vaccine. New England Journal of Medicine. 2020.

7. Public Health England. Technical summary: Public Health England data series on deaths in people with COVID-19 2020 [Available from:

https://www.gov.uk/government/publications/phe-data-series-on-deaths-inpeople-with-covid-19-technical-summary.

8. NHS Digital. COVID-19 - high risk shielded patient list identification methodology 2020 [Available from: https://digital.nhs.uk/coronavirus/shieldedpatient-list/methodology/rule-logic.

9. Public Health England. Public Health England vaccine effectiveness report - March 2021: Public Health England; 2021 [Available from: https://www.gov.uk/government/publications/phe-monitoring-of-theeffectiveness-of-covid-19-vaccination.

10. Haas EJ, Angulo FJ, McLaughlin JM, Anis E, Singer SR, Khan F, et al. Nationwide Vaccination Campaign with BNT162b2 in Israel Demonstrates High Vaccine Effectiveness and Marked Declines in Incidence of SARS-CoV-2 Infections and COVID-19 Cases, Hospitalizations, and Deaths. 2021. 